\title{
Nonlocal fermions and the entropy volume law
}

\author{
G. C. Levine \\ Department of Physics and Astronomy, Hofstra University, Hempstead, NY 11549
}

(Dated: January 17, 2019)

\begin{abstract}
To produce a fermionic model exhibiting an entanglement entropy volume law, we propose a particular version of nonlocality in which the energy-momentum dispersion relation is effectively randomized at the shortest length scales while preserving translation invariance. In contrast to the ground state of local fermions, exhibiting an entanglement entropy area law with logarithmic corrections, the entropy of nonlocal fermions is extensive, scaling as the volume of the subregion and crossing over to the anomalous fermion area law at scales larger than the locality scale, $\alpha$. In the 1-d case, we are able to show that the central charge appearing in the universal entropy expressions for large subregions is simply related to the locality scale. These results are demonstrated by exact diagonalizations of the corresponding discrete lattice fermion models. Within the Ryu-Takayanagi holographic picture, the relation between the central charge and the locality scale suggest a dual spacetime in which the size of the flat UV portion and the radius of AdS in the IR are both proportional to the locality scale, $\alpha$.
\end{abstract}

PACS numbers:

\section{INTRODUCTION}

Nonlocal discrete lattice models and field theories have attracted attention recently in several-possibly related-subfields of physics, spanning quantum information, condensed matter and high energy physics. Random spin models such as [1] have been introduced in connection with the phenomenology of strange metals, but also appear to be important in constructing exactly solvable quantum models with gravitational duals [2]. Nonlocal models have also appeared in the study of the thermalization hypothesis providing the rigorous basis of the canonical ensemble of statistical mechanics [3]. Attempts to understand the internal degrees of freedom of black holes, consistent with the no-cloning theorem, have led to the notion of "fast-scrambling" 4, 5] which also can be realized with certain nonlocal models [6, 7]. So far the fermionic and spin models considered either explicitly break translation invariance or simply do not have spatial degrees of freedom in the conventional sense.

This work attempts to extend nonlocality to fermionic models while maintaining translation invariance. The Maximally Entangled Renormalization Ansatz (MERA) provides a basis for the construction of hamiltonians with a prescribed long distance entanglement law. Motivated by the continuous version of MERA [8, Li and Takayanagi [9, 10] proposed a nonlocal action of the form

$$
S=\int d^{d} x \phi(x) e^{-\alpha \sqrt{\partial^{\mu} \partial_{\mu}}} \phi(x)
$$

where $\alpha$ may be thought of as the scale of nonlocality.

However, the extension to fermions poses a difficulty seen in the following free (non-relativistic) fermion hamiltonian:

$$
H=\epsilon \int d x \psi^{\dagger}(x) e^{-\frac{1}{2} \alpha^{2} \partial_{x}^{2}} \psi(x)
$$

Considering the corresponding discrete model on a periodic lattice of $R$ sites, $H=\sum_{x y} c_{x}^{\dagger} e^{S_{x y}} c_{y}$ (see eq. 110), the nonlocal energy dispersion is $\epsilon_{n}=\epsilon e^{-\alpha^{2} \cos k_{n}}$ where the wavenumber $k_{n}=2 n \pi / R$ and $n, x \in\left[-\frac{R}{2}, \frac{R}{2}\right]$. The zero temperature correlation functions of the half occupied band, $G(x)=\left\langle c_{x}^{\dagger} c_{0}\right\rangle$ may be written:

$G(x)=\frac{1}{R} \sum_{n=-R / 2}^{R / 2} \theta\left(\epsilon_{\mathrm{F}}-\epsilon_{n}\right) e^{i k_{n} x}=\frac{1}{R} \sum_{n=-R / 2}^{R / 2} \theta\left(-k_{n}\right) e^{i k_{n} x}$

where $\theta$ is the unit step function and the Fermi energy, $\epsilon_{\mathrm{F}}=\epsilon$.

Since the nonlocal energy dispersion function preserves wavenumber order of the eigenvalues that is identical to the conventional dispersion $\left(\epsilon_{n}=-\cos k_{n}\right)$, the correlation functions in local and nonlocal cases (equations (3)) are also identical at zero temperature. Entanglement entropy may be computed by a a standard procedure 11] from lattice correlation functions for noninteracting fermions, thus nonlocality in the hamiltonian above is not expected to produce a different entropy area law for fermions. In contrast to the last relation in equation (3), boson correlation functions explicitly involve the dispersion relation and therefore reflect the nonlocality of the kinetic energy in (1).

To overcome this difficulty, we propose models of the following type:

$$
H=\epsilon \int d x \psi^{\dagger}(x) \cos \left(i \alpha \partial_{x}\right) \psi(x)
$$

As in (1) and (2) the kinetic energy operator involves derivatives of all orders and nonlocality. However, If $\alpha / \pi$ is large and irrational, nearby energy states below the Fermi level will be drawn from widely disparate wavenumbers - a feature we refer to as compact nonlocality: maintaining the energy volume of the Fermi sea but scrambling the energy order of the translation invariant eigenfunctions.

This model has two interesting features. First, $\alpha \rightarrow 0$ reproduces nonrelativistic fermions with a quadratic dis- 
persion. Second, in the opposite limit $\alpha \rightarrow \infty$, the irrational values of $\alpha / \pi$ lead to random correlation functions resembling those of models with quenched disorder, although the present hamiltonian is translation invariance. This is the specific feature leading to the volume entropy law.

Quantum many body states exhibiting an entropy volume law were first realized as excited states of integrable hamiltonians 12 and some interesting aspects deeply explained in [13] (see also [14]). Viewed this way, the present work may bear some relation to this earlier work in that the ground state of our model for a given locality parameter $\alpha$ must correspond to a particular excited state of the corresponding local model. Recently there has also been interesting work on constructing exotic spin models exhibiting an entropy volume law in their ground state [15, 16. As we only consider realizations of the hamiltonian 4 on a lattice, this work represents only one possible approach to a constructing a theory with a volume law for fermions. Much analytical work has been accomplished on nonlocal QFT and flat space holography ([17 19]) and it is quite possible that there are other translationally invariant approaches to nonlocal fermions.

\section{NONLOCAL FERMIONIC MODELS}

Nonlocal bosons defined by (1) have been shown analytically 9] and numerically [10] to possess a volume entropy law. As mentioned in the introduction, the difference between boson and fermion behaviors may be traced to the presence of the dispersion relation in the correlator, a feature of relativistic bosons. Real space field operators have particle and antiparticle contributions: $\phi(x) \sim \sum \sqrt{\frac{1}{\omega_{k}}}\left(a_{k}+a_{-k}^{\dagger}\right) e^{i k x}$ where the Bogolubov coefficients explicitly involve the energy dispersion. Fermionic superconductors share this feature with relativistic bosons, having real space field operators that mix particles and antiparticle components and a relativistic dispersion relation with the superconducting gap playing the (momentum dependent) role of particle mass. To this end it is natural to try to construct a model of nonlocal fermions by applying nonlocal extensions to fermionic superconductors. We will show, however, that the problems associated with nonlocal fermions described in the introduction persist even in the relativistic (superconducting) model and necessitate compact nonlocal fermionic extensions. To set the notation used in this manuscript it is convenient to start with the superconducting model before discussing models akin to (4).

Consider a one-dimensional pairing hamiltonian for spinless fermions:

$$
H=\sum_{x, y=0}^{R-1}\left(c_{x}^{\dagger} A_{x y} c_{y}+\frac{1}{2}\left[c_{x}^{\dagger} B_{x y} c_{y}^{\dagger}-c_{x} B_{x y} c_{y}\right]\right)
$$

where $x$ and $y$ are one-dimensional site indices and $A_{x y}$ and $B_{x y}$ are real kinetic energy and pairing matrices respectively, obeying $A_{x y}=A_{y x}$ and $B_{x y}=-B_{y x}$. the operators $c_{x}\left(c_{x}^{\dagger},\right)$ destroys (creates) fermions at site $x$ of a periodic $R$ site lattice and obey the conventional fermion algebra. Applying a Bogoliubov transformation

$$
\begin{aligned}
\eta_{n} & =\sum_{x=0}^{R-1}\left(u_{n x} c_{x}+v_{n x} c_{x}^{\dagger}\right) \\
\eta_{n}^{\dagger} & =\sum_{x=0}^{R-1}\left(u_{n x} c_{x}^{\dagger}+v_{n x} c_{x}\right)
\end{aligned}
$$

brings (5) into diagonal form:

$$
H=\sum_{n} E_{n} \eta_{n}^{\dagger} \eta_{n}
$$

where $\left\{u_{n x}\right\}$ and $\left\{v_{n x}\right\}$ are real coefficients, satisfying a lattice form of the Bogoliubov-de Gennes equation:

$$
\left(\begin{array}{cc}
\mathbf{A} & \mathbf{B} \\
-\mathbf{B} & -\mathbf{A}
\end{array}\right)\left(\begin{array}{l}
\vec{u}_{n} \\
\vec{v}_{n}
\end{array}\right)=E_{n}\left(\begin{array}{l}
\vec{u}_{n} \\
\vec{v}_{n}
\end{array}\right)
$$

The notation in (9) has been simplified by writing the coefficients $\left\{u_{n x}\right\}$ and $\left\{v_{n x}\right\}$ as real space vectors, referenced by energy index $n$. Defining symmetric and antisymmetric lattice derivatives:

$$
\begin{aligned}
& S_{x y}=\frac{1}{2}\left(\delta_{x, y+1}+\delta_{x, y-1}\right) \\
& T_{x y}=\frac{1}{2}\left(\delta_{x, y+1}-\delta_{x, y-1}\right)
\end{aligned}
$$

The conventional dispersion relation of superconducting quasiparticles is obtained by choosing nearest neighbor kinetic and pairing matrices, $A_{x y}=S_{x y}$ and $B_{x y}=T_{x y}$ :

$$
E_{n}^{2}=t_{n}^{2}+\Delta_{n}^{2}
$$

where $t_{n} \equiv \cos (2 \pi n / R)$ and $\Delta_{n} \equiv \sin (2 \pi n / R)$. We will refer to this as the local relativistic dispersion relation. Although other choices will be discussed at the end of this section we presently choose matrices for $\mathbf{A}$ and $\mathbf{B}$ that are symmetric and antisymmetric, respectively, and have compact nonlocality features.

$$
\begin{aligned}
& A_{x y}=[\cos (\alpha \mathbf{S})]_{x y}=[\cos \tilde{\partial}]_{x y} \\
& B_{x y}=[\sinh (-\alpha \mathbf{T})]_{x y}=-i[\sin \partial]_{x y}
\end{aligned}
$$

With the definitions $\tilde{\partial} \equiv \alpha \mathbf{S}$ and $\partial=i \alpha \mathbf{T}$, these matrices satisfy $\partial^{\dagger}=\partial$ and $\tilde{\partial}^{\dagger}=\tilde{\partial}$, and the nonlocal Bogoliubov de Gennes equation may written compactly:

$$
\left(\begin{array}{cc}
\cos \tilde{\partial} & -i \sin \partial \\
i \sin \partial & -\cos \tilde{\partial}
\end{array}\right)\left(\begin{array}{l}
\vec{u}_{n} \\
\vec{v}_{n}
\end{array}\right)=E_{n}\left(\begin{array}{c}
\vec{u}_{n} \\
\vec{v}_{n}
\end{array}\right)
$$

or

$$
\begin{gathered}
H \vec{\phi}_{n}=E_{n} \vec{\phi}_{n} \\
H=\sigma_{z} \cos \tilde{\partial}+\sigma_{y} \sin \partial
\end{gathered}
$$


The energy dispersion now exhibits compact nonlocality in both kinetic and gap (mass) components:

$$
E_{n}^{2}=\cos ^{2}\left(\alpha t_{n}\right)+\sin ^{2}\left(\alpha \Delta_{n}\right)
$$

We now turn to models without pairing, and in particular, those that reduce to nonrelativistic lattice fermions in the local limit $\alpha \rightarrow 0$. As mentioned in the Introduction, eq. (4) reduces to nonrelativistic fermions with quadratic dispersion as $\alpha \rightarrow 0$. with $\epsilon=1 / \alpha^{2}$ identified as the nonrelativistic localization energy scale of a mass $m$ particle: $\hbar^{2} / 2 m \alpha^{2}$ (and $\hbar^{2} / 2 m=1$ ). Using eq. 10 , the lattice form of equation (4) may be written:

$$
H=\epsilon \sum_{x y} c_{x}^{\dagger}[\cos \partial]_{x y} c_{y}
$$

Extending the definition of the symmetric lattice derivative, $S_{x y}$, to higher dimensions, the following hamiltonian has a quadratic dispersion local limit $(\alpha \rightarrow 0)$ in any spatial dimension:

$$
H=\epsilon \sum_{x y} c_{x}^{\dagger}[\sin \tilde{\partial}]_{x y} c_{y}
$$

In the next section entanglement entropy for variations of these models will be explored and compared.

\section{NUMERICAL RESULTS}

Following the procedure developed by Peschel [1] we compute the von Neumann entropy for various nonlocal, quadratic fermion models based upon hamiltonians (5. 12 , (17) and (18). For computing the entropy, the relevant greens functions are

$$
\begin{aligned}
G_{x y} & \equiv\left\langle c_{x}^{\dagger} c_{y}\right\rangle=\sum_{n} v_{n x} v_{n y} \\
F_{x y} & \equiv\left\langle c_{x}^{\dagger} c_{y}^{\dagger}\right\rangle=\sum_{n} v_{n x} u_{n y}
\end{aligned}
$$

where the coefficients $\left\{u_{n x}\right\}$ and $\left\{v_{n x}\right\}$ were found by diagonalizing the BdG hamiltonian (9). The entanglement entropy of a subregion is then computed by extracting the $\mathbf{G}$ and $\mathbf{F}$ matrices for the subregion and computing the entanglement eigenvalues $\left\{\epsilon_{l}\right\}$ from

$$
\begin{aligned}
\sum_{x y}\left(G_{z x}-F_{z x}\right. & \left.-\frac{1}{2} \delta_{z x}\right)\left(G_{z x}+F_{z x}-\frac{1}{2} \delta_{z x}\right) P_{l y} \\
& =\frac{1}{4} \tanh ^{2} \frac{\epsilon_{l}}{2} P_{l z}
\end{aligned}
$$

Finally, the entropy is given by:

$$
S=\sum_{l}\left(\log \left(1+e^{-\epsilon_{l}}\right)+\frac{\epsilon_{l}}{1+e^{\epsilon_{l}}}\right)
$$

We first address the issue of the different possible forms of nonlocality for fermions. As a baseline, the entanglement entropy for a 1-d system described by the local pairing hamiltonian (5) is computed in the gapped phase with kinetic and pairing matrices, respectively, given $\mathbf{A}=\mathbf{S}$ and $\mathbf{B}=\mathbf{T}$. As expected, the entropy $S \sim O(1)$ as shown for a 100 site lattice in figure1. Next we consider a model with nonlocality similar to the model introduced by Shiba and Takayanagi [10, (our equation (1)), but possessing the relativistic dispersion characteristic of a superconductor. In hamiltonian (5) we choose $\mathbf{A}=\cosh (\alpha \mathbf{S})$ and $\mathbf{B}=\sinh (\alpha \mathbf{T})$. The entropy (figure 1) is seen to saturate at a scale much shorter than the locality scale $\alpha=30$. Finally we compute the entropy for a model with compact nonlocality given by hamiltonian (5) and choosing the kinetic and pairing matrices by equations 12 . For the case of $\alpha=30$, the entropy shows a linear regime extending approximately to the locality scale and saturating due to finite size effects at larger scales. Looking at $\alpha=5$ (and $\alpha=15$ ) it appears that the crossover to saturated behavior occurs at $L \approx \alpha$.

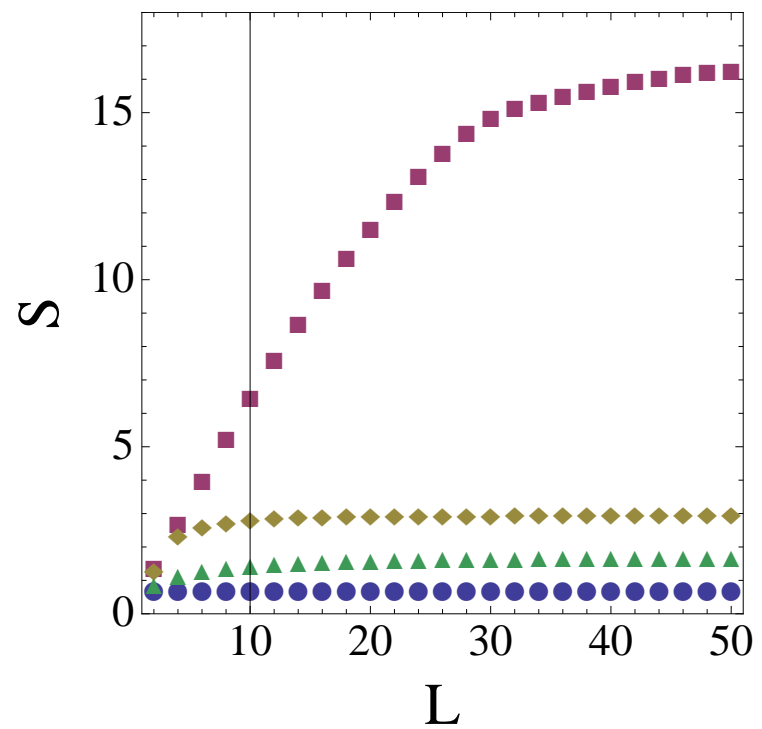

FIG. 1: Entropy $(S)$ versus length $(L)$ of a subregion for several local and nonlocal models on a one-dimensional, 100site lattice. Entropy computed for local, gapped superconducting hamiltonian (equation 5) with kinetic and pairing matrices given by $\mathbf{A}=\mathbf{S}$ and $\mathbf{B}=\mathbf{T}$ (equations 10 ( (०). Entropy computed for noncompact, nonlocal hamiltonian (5) with $\mathbf{A}=\cosh (\alpha \mathbf{S})$ and $\mathbf{B}=\sinh (\alpha \mathbf{T})$ and $\alpha=30(\triangle)$. Entropy computed for compact nonlocal hamiltonian (5), choosing the kinetic and pairing matrices by equations $112 ; \alpha=5$ $(\diamond) ; \alpha=30(\square)$. For $\alpha=30$, the entropy shows a linear regime extending approximately to the locality scale and saturating due to finite size effects at larger scales.

Our conclusion is that nonlocality of a noncompact form in a fermionic hamiltonian with relativistic dispersion does not produce an entropy volume law, despite its similarities to relativistic bosonic models. However, compact nonlocality appears to produce the desired entropy volume law.

Because a relativistic dispersion, analogous to bosons, does not appear to be a necessary feature for a volume entropy law, we now turn to the simpler variants of nonlo- 
cal fermions described by hamiltonians 17 and 18 . For reference, the entropy is computed for a one-dimensional local (nearest neighbor hopping) model of spinless lattice fermions given by the hamiltonian

$$
H=\sum c_{x}^{\dagger} S_{x y} c_{y}
$$

Figures 2 and 3 show the characteristic logarithmic behavior for the entropy of 1-d gapless fermions; for the lower curve, the entanglement entropy is found numerically to follow: $S=c_{0}+\left(c_{\text {eff }} / 3\right) \log L$, where $c_{0}$ is a constant and $c_{\text {eff }} \approx 0.978$ close to the expected value of the central charge $c=1$.

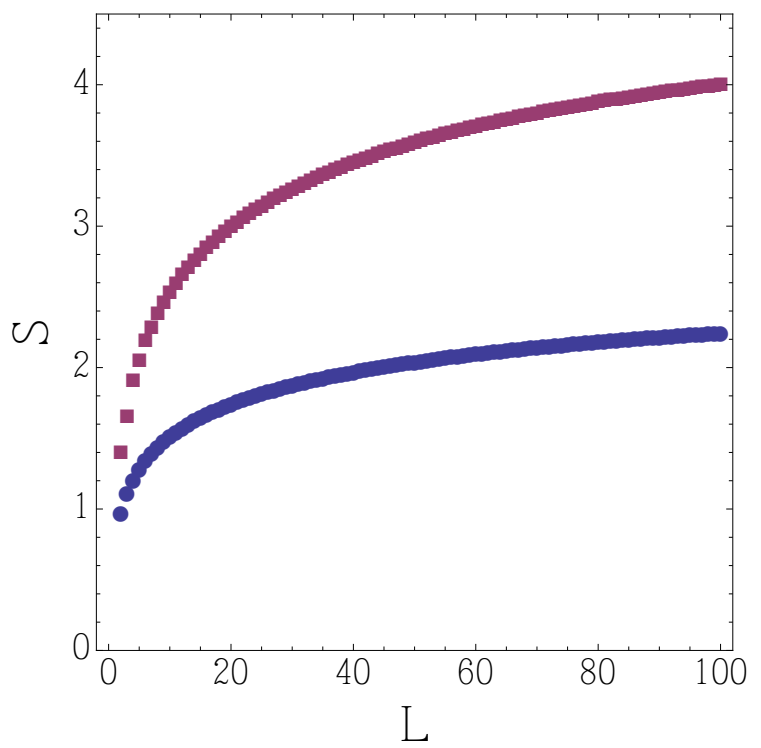

FIG. 2: Entanglement entropy, $S$, of size $L$ subregion computed for the local fermion hamiltonian (23) on a 400 site 1-d lattice with an occupancy fraction $f=$ \#particles/\#sites $=1 / 2(\circ)$. The effective central charge is computed to be $c_{\text {eff }}=0.978 \approx 1$. Corresponding computation for nonlocal hamiltonian (17) with $\alpha=0.01(\square)$.

Next, we compute the entropy for hamiltonian (17) in the local limit $\alpha \rightarrow 0$. Even though (17) appears to be the lattice limit of hamiltonian (4)-i.e. the matrix $i[\partial]_{x y}=$ $T_{x y}$ is the lattice first derivative - it is well known that this model does not express a single chiral lattice fermion. Specifically, it exhibits two fermi points at $k=0, \pi$, and therefore has two quasi-independent fermion species leading to a $c=1$ theory. Diagonalizing this model for small $\alpha$ is in effect diagonalizing $H=\sum c_{x}^{\dagger}\left[\mathbf{T}^{2}\right]_{x y} c_{y}$ or equivalently, $H=\sum c_{x}^{\dagger}\left[\mathbf{S}^{2}\right]_{x y} c_{y}$. Entropy as a function of subsystem length is computed for hamiltonian (17) with $\alpha=0.01$ and displayed in figures 2 and 3 . The behavior is logarithmic: $S \approx\left(c_{\text {eff }} / 3\right) \log L$ with $c_{\text {eff }}=1.96 \approx 2$.

The dependence of the entropy upon the locality scale $\alpha$ in the nonlocal hamiltonian (17) is studied in figure 4. Looking at the large $\alpha$ cases first, there appears to be a nearly linear regime where the entropy behaves as $S \approx d L$ for $L \ll \alpha$ with $d \approx 0.5$. Analysis of the $L>\alpha$

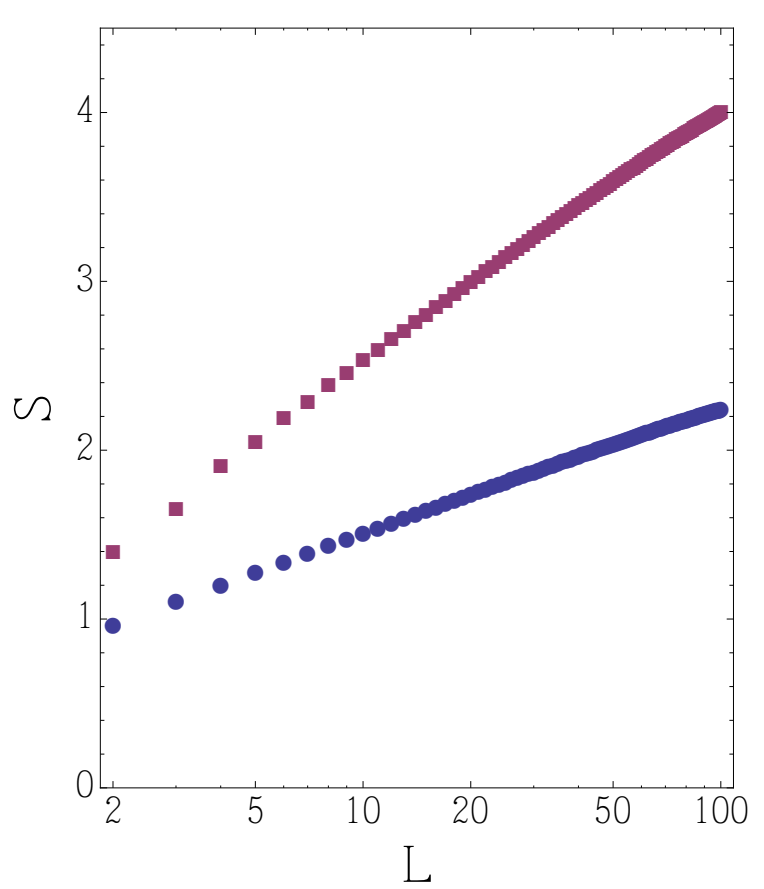

FIG. 3: Log-linear plot of data from figure 2

regime is displayed on the log-linear plot of the same data in figure 5. For this nonlocal model, there appears to be logarithmic regime, for $L>\alpha$, described approximately by $S \approx\left(c_{\text {eff }} / 3\right) \log L$. The factor $c_{\text {eff }}$, which may associated with the number of fundamental degrees of freedom, is seen to be approximately the same as the locality parameter: $c_{\mathrm{eff}} \approx 1.17 \alpha$. This feature will be discussed in the next section.

To extend these computations to 2-d square lattices, hamiltonian $(18)$ is used with the lattice differential operator $S_{\mathbf{x}, \mathbf{y}}=\delta_{\langle\mathbf{x}, \mathbf{y}\rangle}$ where $\langle\mathbf{x}, \mathbf{y}\rangle$ denotes all possible nearest neighbor 2 -d lattice vector points $\mathbf{x}$ and $\mathbf{y}$. The choice of the symmetric matrix, $\mathbf{S}$, avoids cross terms in the higher derivatives of the $\sin \partial$ expansion, such as $\partial_{x_{1}} \partial_{x_{2}}$ where $x_{1}$ and $x_{2}$ are orthogonal lattice directions. With this choice, the discrete derivative operator in hamiltonian (18) may be thought of as the lattice version of $\sin \nabla^{2}$

Using a $61 \times 61$ site periodic lattice, the entanglement entropy was computed for a series of square subregions of linear size $L$. Figure 6 shows the results for several locality factors, $\alpha$. The computations for large $\alpha$ show a $L<<\alpha$ regime where the entropy appears to follow a volume law proportional to $L^{2}$, with a prefactor similar to the 1 -d case: $S \approx d L^{2}$ where $d \approx 0.5$. The behavior in the vicinity of $L \sim \alpha$ is best seen in figure 7; for $L>\alpha, S / L$ clearly becomes sub-linear. Examining $S / L$ on a $\log$-linear plot (figure 8) suggests that $S \propto L \log L$ for $L>\alpha$, a crossover to the anomalous fermion area law 20. To analyze this behavior, we note that the entropy of local model is known to follow the following exact an- 


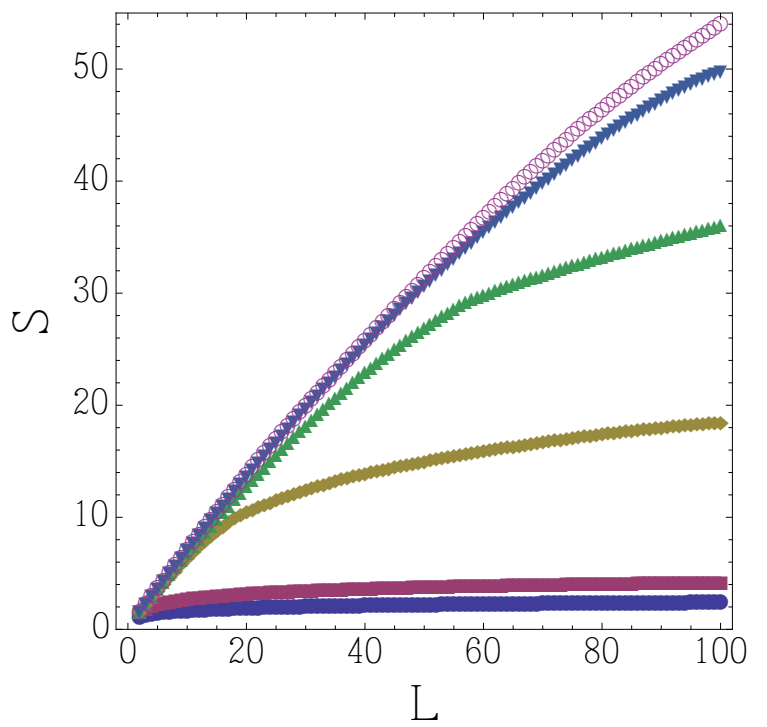

FIG. 4: The dependence of the entropy upon the locality scale $\alpha$ in the compact nonlocal hamiltonian (17). Entropy $S$ versus subregion size $L$ for local models from figure 2 as a reference (solid $\circ$ and solid $\square$ ). $S$ versus $L$ computed for hamiltonian (5) on a 400-site periodic 1 -d lattice, with $\alpha=10,30,50,1400$ $(\diamond, \triangle$, inverted $\triangle, \circ)$. For subregions much smaller than the locality scale $(L \ll \alpha)$, approximately linear behavior is seen.

alytic form: $S=(2 / 3) c_{\text {eff }} L \log L\left[21,22\right.$, where $c_{\text {eff }}=1$ anticipating the relationship of the 2-d anomalous area law to the underlying 1-d tomographic fermions [23, 24]. Computational limitations restrict our computations to sizes no bigger than $61 \times 61$, thus saturation of the entropy appears at subregions of size $L \approx 30$. However, for locality factors between $\alpha=5$ and $\alpha=15$ there is enough data between before saturation to reliably fit $S / L$ logarithmically and determine:

$$
S \approx \frac{2}{3} c_{\mathrm{eff}} L \log L
$$

where $c_{\mathrm{eff}} \approx 1.26 \alpha$, similar to the relationship in 1-d: $c_{\text {eff }}=1.17 \alpha$. In the following section we will discuss the identification of $\alpha$ with the number of fundamental degrees of freedom and the relevance of these fits of 1-d and 2-d entropy data.

In summary, we find the entropy dependence upon linear subregion size $(L)$ for the nonlocal 1-d hamiltonian (17) to be:

$$
\begin{aligned}
& S=A L \quad(L<\alpha) \\
& S=A \alpha+B \frac{\alpha}{3} \log L \quad(L>\alpha)
\end{aligned}
$$

and for the nonlocal 2-d hamiltonian (18):

$$
\begin{aligned}
& S=A L^{2} \quad(L<\alpha) \\
& S=A \alpha^{2}+B^{\prime} \frac{2 \alpha}{3} L \log L \quad(L>\alpha)
\end{aligned}
$$

where $A \approx 0.5, B \approx 1.17$ and $B^{\prime} \approx 1.26$.

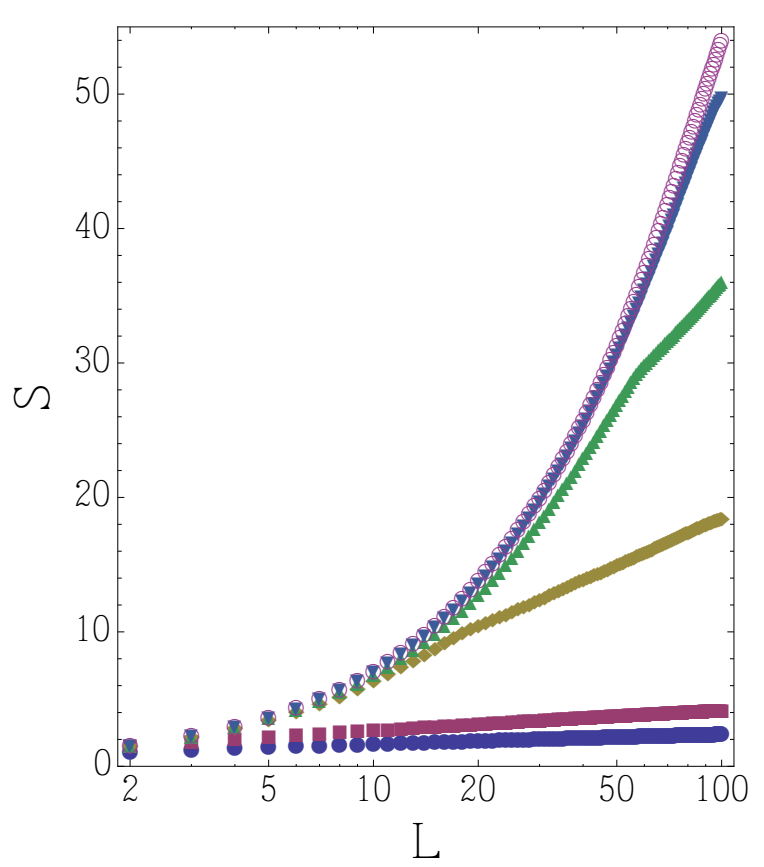

FIG. 5: Log-linear plot of data from figure 4. For subregions much larger than the locality scale $(L>>\alpha)$, entropy depends logarithmically upon $L: S \approx\left(c_{\text {eff }} / 3\right) \log L$ where the effective central charge $c_{\text {eff }} \approx 1.17 \alpha$.

\section{DISCUSSION}

We would like to understand the features of compact nonlocal models that have appeared so far: (1) emergence of the locality parameter, $\alpha$, as a measure of the number of fundamental degrees or effective central charge, $c_{\mathrm{eff}} \approx$ $\alpha(2)$ a conventional fermionic area law for regions $L \gg \alpha$ and (3) the volume entropy law for length scales shorter the locality length $(L \ll \alpha)$.

Consider hamiltonian (4) on a periodic interval $[0, R]$ (and setting the energy scale, $\epsilon=1$ ):

$$
H=\sum_{x y} c_{x}^{\dagger}[\cos \partial]_{x y} c_{y}
$$

The energy spectrum of the model is given by $E_{n}=$ $\cos \left(\alpha \sin k_{n}\right)$, where $k_{n}=2 n \pi / R$ and $n \in[-R / 2, R / 2]$. This model has low energy single particle states with linear dispersion in the vicinity of $\alpha \sin \frac{2 \pi n}{R} \approx \frac{2 m+1}{2} \pi$ where $m=0, \pm 1, \pm 2 \ldots$. Much like the conventional (local) 1-d spinless fermion model, these low energy sectors are independent and each contribute an additive entropy that is logarithmic in the subsystem size. Since $n$ itself is bounded by $\pm R / 2$, the number of low energy domains may be estimated to be: $m_{\max } \approx 4 \alpha / \pi$. The low energy points of $E_{n}$ come in pairs, dispersing with positive and negative velocity; assigning a central charge $c=1 / 2$ to each point give an effective central charge for the nonlocal model $c_{\text {eff }} \approx 2 \alpha / \pi$, similar to our numerical estimates 


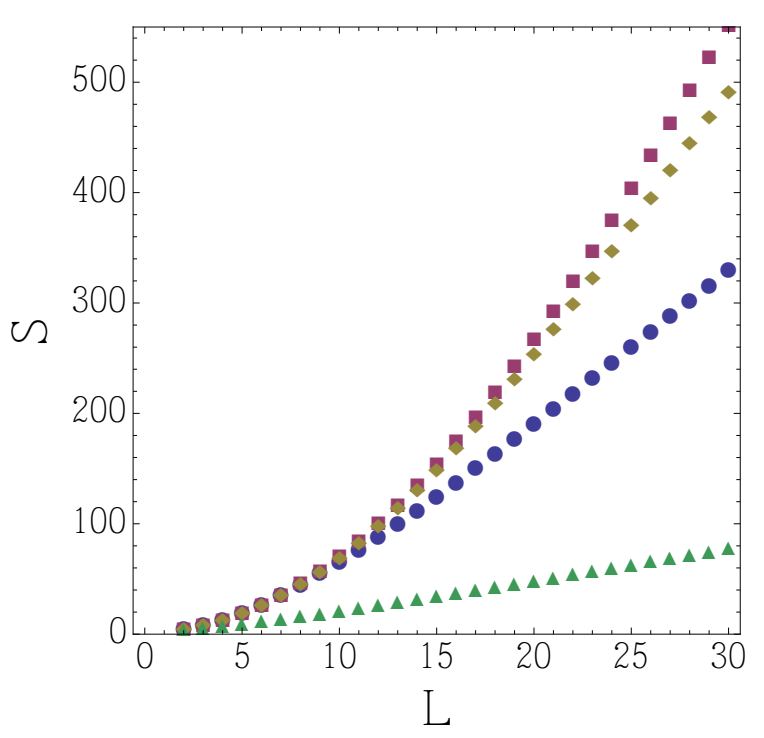

FIG. 6: The entanglement entropy computed for compact nonlocal model hamiltonian 18 extended to 2 -d as described in the text. Dependence of entropy, $S$, on linear size, $L$, of square subregion for a $61 \times 61$ site periodic lattice, computed for several locality parameters, $\alpha$. $S$ versus $L$ for local 2 -d gapless fermions $(\triangle), \alpha=5(\circ), \alpha=15(\diamond), \alpha=1400(\square)$ . The computations for large $\alpha$ show a $L \ll \alpha$ regime where the entropy appears to follow a volume law proportional to $L^{2}$, with a prefactor similar to the 1 -d case: $S \approx d L^{2}$ where $d \approx 0.5$.

from figure 5. Regarding $m$ as a "flavor" index, low energy excitations at a momentum $k_{m}$, given implicitly by $\sin k_{m} \approx \frac{m \pi}{\alpha}$, define a wavepacket of size $\xi \gg \alpha$. To create wavepackets with $\xi \ll \alpha$ involves mixing momentum states over a range $O(1 / \alpha)$, that is, mixing states over two or more adjacent flavor indices. Thus we expect the large $L$ logarithmic behavior to cross over to some other behavior when $L \approx \alpha$.

In the bosonic nonlocal models proposed by $\mathrm{Li}$ and Takayanagi [9], the entropy was computed by a replica/orbifold technique or by directly investigating the structure of the exact reduced density matrix [10. For the model of compact nonlocal fermions presented here, we have not been able to compute the entropy analytically. However, we are able to infer the volume law entropy from properties of the single particle correlation function in the limit of strong nonlocality: $\alpha \gg R, L$.

Consider the lattice correlation function for $\alpha \gg R$ :

$$
G_{x y}=\frac{1}{R} \sum_{m=0}^{R-1}\left\langle c_{m}^{\dagger} c_{m}\right\rangle e^{2 \pi i m(x-y) / R}
$$

where the Fermi distribution $\left\langle c_{m}^{\dagger} c_{m}\right\rangle$ may be regarded as a random binary string with variance $\sigma_{0}^{2}=1 / 4$. The discrete fourier transform of a random function (normalized as above) is another random function with rescaled variance $\sigma^{2}=\sigma_{0}^{2} /(2 R)$. Computation of the entanglement entropy in a subregion depends upon the eigenvalues of

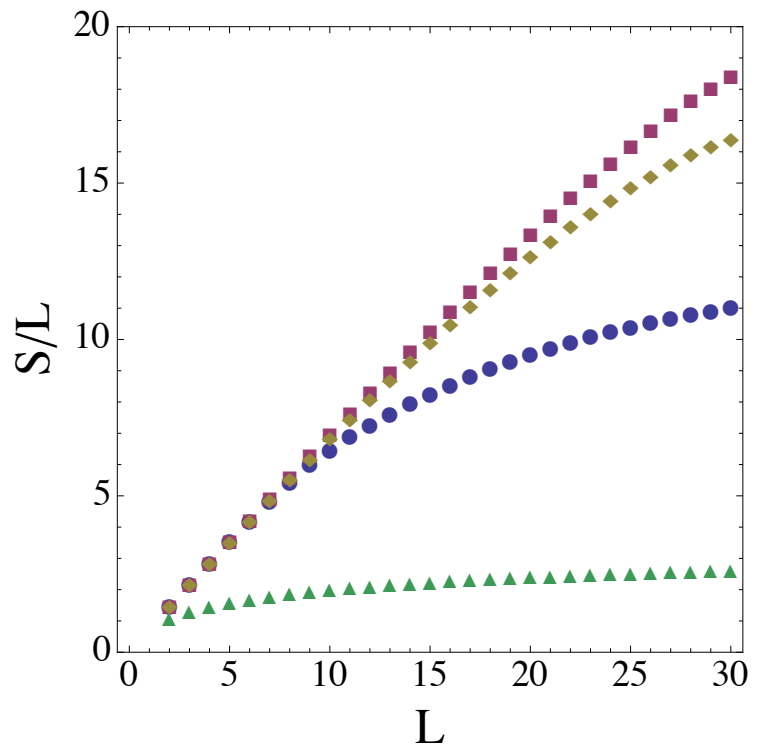

FIG. 7: Data from figure 6 rescaled by $L$. For $L>\alpha$, $S / L$ clearly becomes sublinear but monotonically increasing toward saturation at $L=30$.

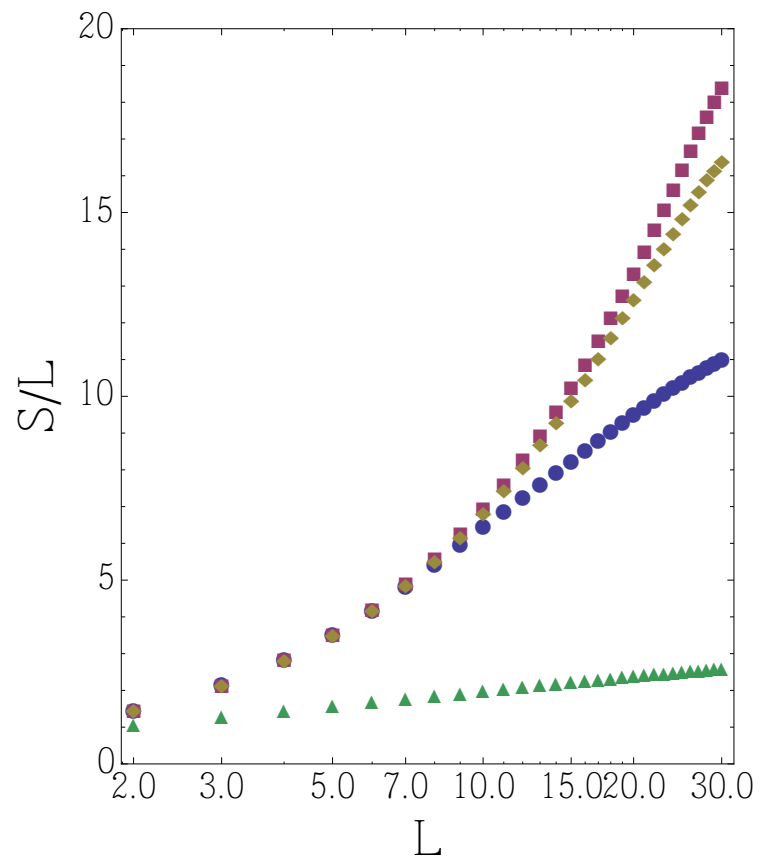

FIG. 8: Data from figure 7 on log-linear plot.

the correlation function restricted to the subregion. The correlation function is a Toeplitz matrix with gaussian distributed random entries. Recently it was proven that such matrices have gaussian distributed random eigenvalues [25]. The von Neumann entropy of $L$ such eigenvalues is proportional to $L$ thus establishing extensive entropy.

Within the holographic picture of Ryu and Takayanagi [26], our central result (equation 25) suggests a bulk 


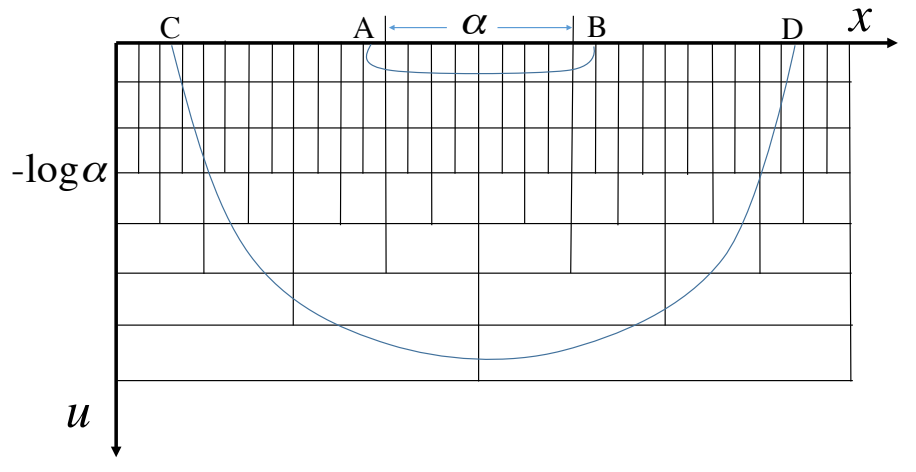

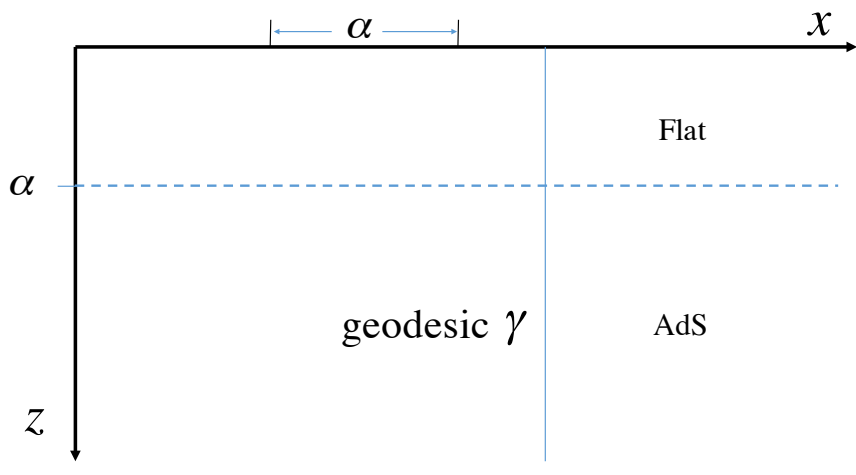

FIG. 9: Cartoon of AdS-flat space hybrid based upon a UV extension of metric 29 to flat space. The entanglement of regions comparable to the locality scale $(L \approx \alpha)$, such as $L_{\mathrm{AB}}$ depicted above, involve geodesics through flat space with minimal extension into the bulk. Regions larger than the locality scale $(L \gg \alpha)$, such as $L_{\mathrm{CD}}$ depicted above, have geodesics that penetrate into the AdS region.

space with a crossover between flat space in the UV and AdS space in the IR to accommodate the crossover in entropy between extensive and logarithmic behaviors as the length of the subregion increases. By the BrownHenneaux relation, the radius of $\mathrm{AdS}_{3}, \kappa$, is proportional to the central charge of the boundary theory, $c=\frac{3 \kappa}{2 G}$, where $G$ is the 3 -d Newton constant. But the effective central charge in our model of compact nonlocality is also proportional to the locality scale, $\alpha$, and the bulk metric must capture this property. Figure 9 is a cartoon of the spatial slice of such a space based upon the structure of the pure $\mathrm{AdS}_{3}$ metric

$$
d s^{2}=\kappa^{2}\left(d u^{2}+\frac{e^{2 u}}{\epsilon^{2}} d x^{2}\right)
$$

showing the space modified by a flat space slice of width $\alpha$ at the boundary. Thus the entanglement of regions shorter than the locality scale $(L \ll \alpha)$ involve geodesics through flat space with minimal extension into the bulk, whereas regions larger than the locality scale $(L \gg \alpha)$ have geodesics that penetrate into the AdS region.

Looking at the behavior of the 1-d entropy (25) for $L$ larger than the locality scale $\alpha$, it is seen that $\alpha$ enters the expression in two additive roles: the saturation of the extensive entropy at the locality scale $L \approx \alpha$ and the dependence of the effective central charge on $\alpha$ in the logarithmic term. Switching to Poincare coordinates in figure 10, consider the vertical portion of the geodesic $\gamma$, appropriate for computing the large $L$ entropy $(L \gg \alpha)$
FIG. 10: Cartoon of AdS-flat space hybrid in Poincare coordinates suggesting the metric (31). The size, in $z$, of the flat space region must be proportional to the locality scale $\alpha$ of the boundary theory, as well as the AdS radius $\kappa \approx \alpha$ to produce the correct entropy-length behavior 25 in the ground state of nonlocal hamiltonian (17).

by the Ryu-Takayanagi construction:

$$
S=\frac{1}{2 G} \int_{\gamma} d s
$$

A critical feature of this geometry is then that the size (in $z$ ) of the flat region must be proportional to the radius of the asymptotic AdS region to reproduce both terms of equation 25) for the entropy: $S=A \alpha+B(\alpha / 3) \log L$. To this end we propose the following spatial slice metric (in Poincare coordinates, $z=\epsilon e^{-u}$ ) capturing these features,

$$
d s^{2}=\tanh \left(\frac{\alpha_{\mathrm{c}}^{2}}{z^{2}}\right)\left(d x^{2}+d z^{2}\right)
$$

where we have replaced the AdS radius with the locality scale, $\alpha_{\mathrm{c}}$. Note that $\alpha_{\mathrm{c}}$ in this expression has dimensions of length corresponding to the continuum hamiltonian (4), and in contrast to the dimensionless $\alpha$ appearing in our lattice computations.

Studying (31) for constant $x$, there is the small $z$ regime $\left(0<z<\alpha_{\mathrm{c}}\right)$ in which $d s \sim d z$, and a large $z$ regime where $d s \sim\left(\alpha_{\mathrm{c}} / z\right) d z$ as $z \rightarrow \infty$. If we consider a large $L$ subregion, the entropy may be approximated by integrating the Ryu-Takayanagi relation along the constant $x$ geodesic $\gamma$ pictured in figure 10 , and imposing an IR cut off of $L$.

$$
\begin{aligned}
S & =\frac{1}{2 G} \int_{0}^{L} d z \sqrt{\tanh \frac{\alpha_{\mathrm{c}}^{2}}{z^{2}}} \\
& \approx \frac{\alpha_{\mathrm{c}}}{2 G}+\frac{\alpha_{\mathrm{c}}}{2 G} \log \frac{L}{\alpha_{\mathrm{c}}}
\end{aligned}
$$




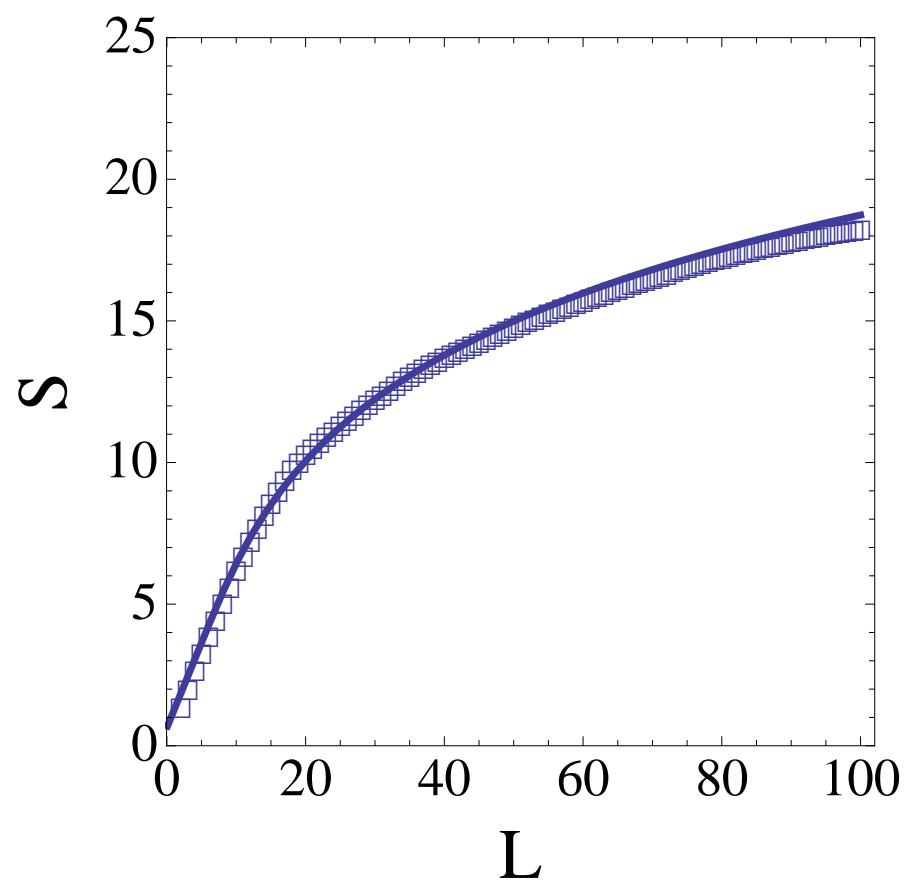

FIG. 11: Numerical integration of length along geodesic $\gamma$ using metric 31 compared with numerical computation of entropy of figure 4, $\alpha=10$. Following equation (32), $S=$ $a \int_{0}^{L} d z \sqrt{\tanh \frac{\alpha_{\mathrm{c}}^{2}}{z^{2}}}+b$ where $\alpha_{\mathrm{c}}=9, a=0.6$ and $b=0.7$

in reasonable agreement with our results for the 1-d entropy 25, making the identification $c_{\text {eff }}=\alpha=\frac{3 \alpha_{c}}{2 G}$. The integral appearing in (32) cannot be done analytically (there is an essential singularity at $z=0$ ) and we resort to a numerical evaluation. Figure 11 provides a comparison of a numerical integration of the geodesic length appearing in equation (32) with our numerical lattice computations for a representative intermediate $\alpha$ case. There is reasonable agreement, but it should be noted that the geodesic $\gamma$ is only an approximation of the putative geodesic corresponding to the finite $L$ lattice computation. Until further studies, we conclude that the proposed metric is a qualitative description of the gravitational dual to the nonlocal model (17).

\section{CONCLUSION AND FURTHER DIRECTIONS}

In this manuscript we have suggested nonlocality for fermions based upon a hamiltonians (17) and (18) incorporating periodic functions of the momentum operator. These models exhibit a crossover between volume and anomalous (logarithmic) area law behavior at scales larger than the locality scale, $\alpha$. The locality scale plays two roles. Firstly, it is the scale at which the volume law saturates. But owing to the periodic nature of the hamiltonian it is also a measure of the number of fun- damental degrees of freedom in the theory - the effective central charge, $c_{\text {eff }}$.

In 1-d we suggest that the hamiltonian (17) has a geometric interpretation within the Ryu-Takayanagi holographic picture of a hybrid AdS space in the IR and Euclidean space in the UV. Because of its dual role, the locality scale determines both the size of the UV flat slice and the radius of the asymptotic AdS space. We propose a specific metric in 3-d bulk space that captures the entropy/length relation for the ground state of this nonlocal model.

There are several obvious directions for future work. Clearly, it would be desirable to compute the entropy analytically for the continuum version of our nonlocal model (4) on an orbifold, parallel to the calculation of [9] for a nonlocal scalar QFT. For our model, note that a bosonized action only corresponds to set of $\left(c_{\text {eff }}\right)$ linearized fermion modes; the nonlocal, extensive, behavior comes from the part of the dispersion between the Fermi points. Thus a replica/orbifold computation involves computing the spectrum of the nonlocal Dirac type operator $\cos \left(i \alpha \partial_{x}\right)$. It may also be interesting to investigate whether a compact dispersion in a scalar boson model analogous to 9] also exhibits a volume law, and whether the locality scale also proportional to the effective central charge.

The combination $i \alpha \partial_{x}$ in the nonlocal hamiltonian (4) also invites the following anisotropic generalization of in $d$ dimensions,

$$
H=\epsilon \int d^{d} x \psi^{\dagger}(x) \cos \left(i \alpha^{\mu} \partial_{\mu}\right) \psi(x)
$$

Anisotropy comes at the cost of destroying the nonrelativistic, quadratic dispersion limit as $\alpha \rightarrow 0$ in dimensions $d>1$. However it has been pointed out 27] that if the set $\left\{\alpha^{\mu}\right\}$ are extended to form a Clifford algebra $\left\{\alpha^{\mu}, \alpha^{\nu}\right\}=\delta_{\mu \nu}$, the nonrelativistic quadratic limit is maintained. Thus an interesting outcome is that nonrelativistic lattice fermions may be realized as the $\alpha \rightarrow 0$ limit of nonlocal fermions with additional isospin degrees of freedom. To include interactions, one possibility is to exploit the well defined (random) UV behavior of the propagators when $\alpha \rightarrow \infty$ and develop a perturbation expansion in $1 / \alpha$. With interactions, an inhomogeneous locality parameter $\alpha=\alpha(x)$ might also be a suitable variational parameter.

Lastly, there are several, related, nonequilibrium properties that might be explored. In a non-translationally invariant model, the thermalization time scale [3] and closely related scrambling time scale [4, 6) have been computationally studied for nonlocal fermions. It seems likely that subsystems smaller than the locality scale in our model would satisfy the Eigenstate Thermalization Hypothesis (ETH), as do globally nonlocal fermions in the cited studies, because the UV behavior of our correlation functions is effectively disordered. For this reason, it would be natural to study the evolution of entanglement in a variety of nonequilibrium initial conditions $28,33$. 


\section{Acknowledgement}

GL wishes to thank Benjamin Burrington and Adam Durst for many interesting discussions. 
[1] S. Sachdev, Phys. Rev. Lett. 105, 151602 (2010) doi:10.1103/PhysRevLett.105.151602 arXiv:1006.3794 [hep-th]].

[2] J. Maldacena and D. Stanford, Phys. Rev. D 94, no. 10, 106002 (2016) doi:10.1103/PhysRevD.94.106002 arXiv:1604.07818 [hep-th]].

[3] J. M. Magan, "Random free fermions: An analytical example of eigenstate thermalization," Phys. Rev. Lett. 116, no. 3, $030401 \quad$ (2016) doi:10.1103/PhysRevLett.116.030401 arXiv:1508.05339 [quant-ph]].

[4] P. Hayden and J. Preskill, "Black holes as mirrors: Quantum information in random subsystems," JHEP 0709, 120 (2007) doi:10.1088/1126-6708/2007/09/120 arXiv:0708.4025 [hep-th]].

[5] Y. Sekino and L. Susskind, "Fast Scramblers," JHEP 0810, 065 (2008) doi:10.1088/1126-6708/2008/10/065 arXiv:0808.2096 [hep-th]].

[6] J. M. Magan, "Black holes as random particles: entanglement dynamics in infinite range and matrix models," JHEP 1608, 081 (2016) doi:10.1007/JHEP08(2016)081 arXiv:1601.04663 [hep-th]].

[7] B. Swingle, G. Bentsen, M. Schleier-Smith and P. Hayden, "Measuring the scrambling of quantum information," Phys. Rev. A 94, no. 4, 040302 (2016) doi:10.1103/PhysRevA.94.040302 arXiv:1602.06271 [quant-ph]].

[8] J. Haegeman, T. J. Osborne, H. Verschelde and F. Verstraete, "Entanglement Renormalization for Quantum Fields in Real Space," Phys. Rev. Lett. 110, no. 10, 100402 (2013) doi:10.1103/PhysRevLett.110.100402 arXiv:1102.5524 [hep-th]].

[9] W. Li and T. Takayanagi, "Holography and Entanglement in Flat Spacetime," Phys. Rev. Lett. 106, 141301 (2011) doi:10.1103/PhysRevLett.106.141301 arXiv:1010.3700 [hep-th]].

[10] N. Shiba and T. Takayanagi, "Volume Law for the Entanglement Entropy in Non-local QFTs," JHEP 1402, 033 (2014) doi:10.1007/JHEP02(2014)033 arXiv:1311.1643 [hep-th]].

[11] Peschel, I. Journal of Physics A Mathematical General 36, L205 (2003) arXiv:cond-mat/0212631.

[12] V. Alba, M. Fagotti and P. Calabrese, "Entanglement entropy of excited states," J. Stat. Mech. 0910, P10020 (2009) doi:10.1088/1742-5468/2009/10/P10020 arXiv:0909.1999 [cond-mat.stat-mech]].

[13] Lai, H.-H. \& Yang, K. "Entanglement entropy scaling laws and eigenstate typicality in free fermion systems," Physical Review B 91, 081110 (2015) arXiv:1409.1224.

[14] L. Vidmar, L. Hackl, E. Bianchi and M. Rigol, "Volume Law and Quantum Criticality in the Entanglement Entropy of Excited Eigenstates of the Quantum Ising Model," arXiv:1808.08963 [cond-mat.stat-mech].

[15] Zhang, Z., Ahmadain, A. \& Klich, I. "Quantum phase transition from bounded to extensive entanglement entropy in a frustration-free spin chain," arXiv e-print arXiv:1606.07795 (2016) arXiv:1606.07795.

[16] O. Salberger, T. Udagawa, Z. Zhang, H. Katsura, I. Klich and V. Korepin, "Deformed Fredkin Spin Chain with Extensive Entanglement," J. Stat. Mech. 1706, no. 6, 063103 (2017) doi:10.1088/1742-5468/aa6b1f arXiv:1611.04983 [cond-mat.stat-mech]].

[17] S. Kachru, X. Liu and M. Mulligan, "Gravity Duals of Lifshitz-like Fixed Points," Phys. Rev. D 78 (2008) 106005 arXiv:0808.1725 [hep-th]]

[18] A. Bagchi, R. Basu, D. Grumiller and M. Riegler, "Entanglement entropy in Galilean conformal field theories and flat holography," Phys. Rev. Lett. 114, no. 11, 111602 (2015) doi:10.1103/PhysRevLett.114.111602 arXiv:1410.4089 [hep-th]].

[19] D. W. Pang, "Holographic entanglement entropy of nonlocal field theories," Phys. Rev. D 89, no. 12, 126005 (2014) doi:10.1103/PhysRevD.89.126005 arXiv:1404.5419 [hep-th]].

[20] Gioev, D. \& Klich, I. Phys. Rev. Lett. 96, 100503 (2006) arXiv:quant-ph/0504151.

[21] Li, W., Ding, L., Yu, R., Roscilde, T. \& Haas, S. 2006, Physical Review B, 74, 073103, arXiv:quant-ph/0602094

[22] Barthel, T., Chung, M.-C. \& Schollwöck, U. Physical Review A 74, 022329 (2006) arXiv:cond-mat/0602077.

[23] G. C. Levine and D. J. Miller, "Zero-dimensional area law in a gapless fermionic system," Phys. Rev. B 77, 266402 (2008).

[24] B. Swingle, "Entanglement entropy and the Fermi surface," Phys. Rev. Lett. 105, 050502 (2010)

[25] V. Kargin, "Spectrum of random Toeplitz matrices with band structure," Elec. Comm. in Prob. 14 (2009); A. Bose and K. Saha, "Limiting spectral distribution of circulant matrix with dependent entries," unpublished, subsumed in Technical Report R6/2009, Stat-Math Unit, Dept. of Science and Technology, Govt. of India.

[26] S. Ryu and T. Takayanagi, Phys. Rev. Lett. 96 (2006) 181602.

[27] Benjamin Burrington, private communication.

[28] P. Calabrese and J. Cardy, J. Stat. Mech. (2007) P10004.

[29] F. Igloi, Z. Szatmari, and Y.-C. Lin, Phys. Rev. B 85, 094417 (2012).

[30] G. De Chiara, S. Montangero, P. Calabrese and R. Fazio, J. Stat. Mech. 0603:L03001 (2006). cond-mat/0512586

[31] J. M. Stephan and J. Dubail, J. Stat. Mech. (2011) P08019.; U. Divakaran et al, arXiv:1105.5317

[32] Peschel, I. \& Eisler, V. Journal of Physics A Mathematical General 42, 504003 (2009) arXiv:0906.1663.

[33] G. C. Levine, M. Bantegui and J. Burg, "Full counting statistics in a disordered free fermion system," Phys. Rev B 86, 174202 (2012). 